\title{
Integration of matrix-assisted laser desorption/ ionization time-of-flight mass spectrometry in blood culture diagnostics: a fast and effective approach
}

\author{
Correspondence \\ Konrad A. Bode \\ konrad.bode@uni-heidelberg.de
}

Received 23 June 2011

Accepted 3 November 2011

\author{
Sabrina Klein, † Stefan Zimmermann,† Christine Köhler, \\ Alexander Mischnik, Werner Alle and Konrad A. Bode
}

\author{
Department of Infectious Diseases, Medical Microbiology and Hygiene, University of Heidelberg, \\ Im Neuenheimer Feld 324, 69120 Heidelberg, Germany
}

\begin{abstract}
Sepsis is a major cause of mortality in hospitalized patients worldwide, with lethality rates ranging from 30 to $70 \%$. Sepsis is caused by a variety of different pathogens, and rapid diagnosis is of outstanding importance, as early and adequate antimicrobial therapy correlates with positive clinical outcome. In recent years, matrix-assisted laser desorption/ionization time-of-flight (MALDITOF) mass spectrometry (MS) fingerprinting has become a powerful tool in microbiological diagnostics. The direct identification of micro-organisms in a positive blood culture by MALDITOF MS can shorten the diagnostic procedure significantly. Therefore, the aim of the present study was to evaluate whether identification rates could be improved by using the new Sepsityper kit from Bruker Daltonics for direct isolation and identification of bacteria from positive blood cultures by MALDI-TOF MS compared with the use of conventional separator gel columns, and to integrate the MALDI-TOF MS-based identification method into the routine course of blood culture diagnostics in the setting of a microbiological laboratory at a university hospital in Germany. The identification of Gram-negative bacteria by MALDI-TOF MS was significantly better using the Sepsityper kit compared with a separator gel tube-based method (99 and $68 \%$ correct identification, respectively). For Gram-positive bacteria, only $73 \%$ were correctly identified by MALDI-TOF with the Sepsityper kit and $59 \%$ with the separator gel tube assay. A major problem of both methods was the poor identification of Gram-positive grape-like clustered cocci. As differentiation of Staphylococcus aureus from coagulase-negative staphylococci is of clinical importance, a PCR was additionally established that was capable of identifying S. aureus directly from positive blood cultures, thus closing this diagnostic gap. Another benefit of the PCR approach is the possibility of directly detecting the genes responsible for meticillin resistance in staphylococci and for vancomycin resistance in enterococci, which is of high importance for early adequate treatment. Both of the described methods were finally integrated into a protocol for fast and effective identification of bacteria from positive blood cultures.
\end{abstract}

\section{INTRODUCTION}

Sepsis is a major cause of mortality in hospitalized patients worldwide, with lethality rates ranging from 30 to $70 \%$ (Garnacho-Montero et al., 2008). Sepsis can be caused by a variety of different infectious agents. Early and adequate antimicrobial therapy correlates with positive clinical outcomes (Bochud et al., 2004; Ibrahim et al., 2000; Kumar et al., 2006, 2009). Thus, rapid microbiological diagnostics are of high importance to improve the survival of patients.

†These authors contributed equally to this work.

Abbreviations: AST, antimicrobial susceptibility testing; CoNS, coagulase-negative staphylococci; MALDI-TOF, matrix-assisted laser desorption/ionization time of flight; MS, mass spectrometry; PBMC, peripheral blood mononuclear cell; VRE, vancomycin-resistant enterococci.
To date, blood culture has been the gold standard for diagnosis of bloodstream infections. Micro-organisms present in a blood sample are enriched in cultivation medium in blood culture bottles under continuous monitoring. When growth is detected, Gram staining is performed, which allows the first adjustment of antibiotic therapy. Subcultivation is needed for identification and antimicrobial susceptibility testing (AST), which takes on average 12-48 $\mathrm{h}$. Therefore, the results of the final differentiation and AST are often obtained too late to influence vital treatment decisions.

In recent years, matrix-assisted laser desorption/ionization time-of-flight (MALDI-TOF) mass spectrometry (MS) fingerprinting has become a powerful tool in microbiological diagnostics (Emonet et al., 2010). Conventional 
identification of bacteria by subculture and subsequent biochemical reactions takes up to $48 \mathrm{~h}$, depending on the species and system used. In contrast, MALDI-TOF MS can be performed with minimal amounts of bacteria and takes only a few minutes. The direct identification of microorganisms from a positive blood culture can significantly shorten the time for exact species determination, enabling earlier adjustment of the antimicrobial therapy. To date, several methods have been described for the direct identification of micro-organisms from a positive blood culture using MALDI-TOF MS (Christner et al., 2010; Ferreira et al., 2011; Moussaoui et al., 2010; Stevenson et al., 2010).

The aim of the present study was to evaluate the use of a MALDI-TOF MS-based identification method in the routine course of blood culture diagnostics in the setting of a microbiological laboratory at a university hospital. First, the new Sepsityper kit from Bruker Daltonics, developed for the preparation of positive blood cultures for direct MALDI-TOF MS identification, and a separator gel-based method (Moussaoui et al., 2010), were evaluated. From the results obtained in the initial experiments, the workflow for identification of micro-organisms by MALDI-TOF MS was supplemented by a PCR.

\section{METHODS}

Chemicals and media. Cyano-4-hydroxycinnamic acid (HCCA) matrix substance for MALDI-TOF MS measurements and the Sepsityper kit were obtained from Bruker Daltonics. Other chemicals of high purity (HPLC grade) were purchased from Sigma-Aldrich.

Samples. The study was conducted during working hours from May 2010 to December 2010 at the Heidelberg University Hospital. Microbial growth of positive detected culture bottles (BACTEC Plus Aerobic/F, Anaerobic/F and BACTEC Peds Plus/F culture vials, BACTEC 9240 automated blood culture system; BD) was confirmed by Gram staining. Samples showing monoculture in the Gram staining were subjected in parallel to direct identification by MALDITOF MS fingerprinting and conventional subculture for reference identification. The number of blood cultures containing Grampositive grape-like clustered cocci was limited to 100 samples for the separator tube preparation and to 50 samples for the Sepsityper preparation.

Number of analysed positive blood cultures. During the study period, 761 blood cultures were analysed. Overall, 511 bottles were analysed by PCR, 123 samples by the Sepsityper kit and 175 samples by separator tube preparation.

Over the course of the study, all positive blood cultures containing Gram-positive cocci were investigated by a PCR detecting $\mathrm{femB}$ for the identification of Staphylococcus aureus, as well as the resistance genes mecA, vanA and vanB.

Sample preparation using separator tubes. Samples of positive blood culture fluid $(1 \mathrm{ml})$ were centrifuged in a separator gel tube (Vacutainer; BD Diagnostics) for $10 \mathrm{~min}$ at $1500 \mathrm{~g}$ to separate the blood cells and bacteria. The interphase on the top of the gel was washed twice with $1 \mathrm{ml}$ deionized water.

Sample preparation using a Sepsityper kit. Sample preparation using a Sepsityper kit (Bruker Daltonics) was carried out according to the manufacturer's protocol. Briefly, $1 \mathrm{ml}$ blood culture fluid of a positive blood culture was transferred to a reaction tube, $200 \mu$ lysis buffer was added and the tube was vortexed for $10 \mathrm{~s}$. The sample was centrifuged at $18000 \mathrm{~g}$ for $2 \mathrm{~min}$ and the supernatant discarded. The pellet was washed once in $1 \mathrm{ml}$ washing buffer. Finally, the pellet was precipitated by centrifugation $(1 \mathrm{~min}, 18000 \mathrm{~g})$ and the supernatant discarded. If there was no visible pellet after the final centrifugation step, the preparation was repeated with an additional centrifugation step of the blood for $30 \mathrm{~s}$ and $400 \mathrm{~g}$ prior to addition of the lysis buffer. The supernatant was then used to proceed with the preparation as described above.

Protein extraction for MALDI-TOF MS analysis. The isolated bacterial pellet was treated with a standard ethanol/formic acid protein extraction protocol for MALDI-TOF MS identification, with a minor alteration (Mellmann et al., 2009). The bacterial pellet from the extraction step was resuspended in $300 \mu$ l deionized water, the ethanol concentration was adjusted to a concentration of $70 \%(\mathrm{v} / \mathrm{v})$, and the tube was then centrifuged for a second time $(2 \mathrm{~min}$, $18000 \mathrm{~g}$ ). The liquid was removed carefully. To minimize residual ethanol, the samples were centrifuged a second time for $2 \mathrm{~min}$ at $18000 \mathrm{~g}$, the supernatants discarded and the samples left to dry in air for $5 \mathrm{~min}$. Finally, the pellet was resuspended in $25 \mu \mathrm{l} 70 \%(\mathrm{v} / \mathrm{v})$ formic acid and $25 \mu \mathrm{l}$ acetonitrile, and the sample was vortexed briefly. The mixture was centrifuged for $2 \mathrm{~min}$ at $18000 \mathrm{~g}$, and $1 \mu \mathrm{l}$ supernatant was spotted onto a MALDI sample target and air-dried. It was then overlaid with $1 \mu \mathrm{HCCA}$ matrix solution.

After drying of the sample, MALDI-TOF MS analysis was performed on a Bruker Microflex MALDI-TOF mass spectrometer with a nitrogen laser $(337 \mathrm{~nm})$ operating in linear mode with delayed extraction at $20 \mathrm{kV}$ accelerating voltage. Each spectrum was automatically collected in the positive ion mode with a mean of 700 laser shots (50 laser shots at 14 different spot positions). A mass range between 2000 and $20000 \mathrm{~m} / \mathrm{z}$ (ratio of mass/charge) was selected to collect the signals with the AutoXecute tool of the FlexControl acquisition software (version 2.4; Bruker Daltonics). Bacteria were identified using the MALDI BioTyper 2.0 software and the Bruker database V. All MALDI-TOF MS results were compared by identification using standard biochemical methods (Vitek2 system and API system; bioMérieux) and discrepancies were resolved by $16 \mathrm{~S}$ rRNA gene sequencing. The MALDI BioTyper output is a $\log$ (score) in the range 0-3.0, computed by comparison of the peak list for an unknown isolate with the reference mass spectra in the database. A $\log$ (score) of $\geqslant 1.7$ is indicative of a close relationship (i.e. at the genus level) and a $\log$ (score) of $\geqslant 2.0$ is the set threshold for a match at the species level.

DNA extraction directly from positive blood culture bottles. A $500 \mu \mathrm{l}$ sample of the positive detected blood culture fluid was transferred to a reaction tube. The sample was centrifuged at $18000 \mathrm{~g}$ for $1 \mathrm{~min}$ and the supernatant discarded. The pellet was resuspended in $1 \mathrm{ml}$ lysis buffer $[10 \mathrm{mM}$ Tris/ $\mathrm{HCl}(\mathrm{pH} 8), 1 \mathrm{mM}$ EDTA, $1 \%$ Triton X-100] and vortexed for $10 \mathrm{~s}$. After centrifugation at $18000 \mathrm{~g}$ for $1 \mathrm{~min}$, the supernatant was discarded and the pellet washed twice in $500 \mu \mathrm{l}$ washing buffer [ $30 \mathrm{mM}$ Tris/ $\mathrm{HCl}(\mathrm{pH} 8), 3 \mathrm{mM}$ EDTA] by centrifugation at $18000 \mathrm{~g}$ for $1 \mathrm{~min}$. Thereafter, the pellet was resuspended in DNA purification buffer $[30 \mathrm{mM}$ Tris/ $\mathrm{HCl}(\mathrm{pH} 8)$, $3 \mathrm{mM}$ EDTA and 50-100 $\mu$ l glass beads (150-200 $\mu \mathrm{m}$ diameter) per $500 \mu \mathrm{l}$, vortexed for $15 \mathrm{~min}$ and centrifuged for $3 \mathrm{~min}$ at $18000 \mathrm{~g}$. The supernatant was used in a $1: 100$ dilution for the PCR.

PCR. Bacterial DNA was amplified using a double-dye oligonucleotide real-time PCR (SmartCycler; Cepheid) using a commercial reagent kit (ABgene). DNA $(2.5 \mu \mathrm{l})$ was used as template in a $22.5 \mu \mathrm{l}$ PCR mix containing $10 \mathrm{pmol}^{-1}$ of each DNA probe and $50 \mathrm{pmol}$ $\mu \mathrm{l}^{-1}$ of each primer. Cycling conditions were $95{ }^{\circ} \mathrm{C}$ for $10 \mathrm{~min}$, 
followed by 37 cycles of $95{ }^{\circ} \mathrm{C}$ for $15 \mathrm{~s}, 56{ }^{\circ} \mathrm{C}$ for $30 \mathrm{~s}$ and $72{ }^{\circ} \mathrm{C}$ for $30 \mathrm{~s}$. The primers and probes used were: $\operatorname{mec} A$ fw, $5^{\prime}$-GATAAAAAAGAACCTCTGCT-3'; mecA rv, 5'-ACTGCCTAATTCGAGTG-3'; mecA-probe, 5' -FAM-ACAACTTCACCAGGTTCAACTCAAA-BHQ1 -3'; femB fw, 5'-AATTAACGACGAAATGGGCAGAAACA-3'; femB rv, 5' -TGCGCAACACCCTGAACTT-3'; femB-probe, 5' -TET-AGAAATTAACTGGATGGTACGCGCGAAGA-BHQ1-3'; vanB fw, 5'-GGACAAATCACTGGCCTACATTC-3'; vanB rv, 5'-CGCCGACAATCAAATCATC-3'; vanB-probe, 5'-FAM-ACCTACCCTGTCTTTGTGAAGCCG-BHQI-3'; vanA fw, 5' -ATGAATAGAATAAAAGTTGCAATACT-3'; vanA rv, 5'-GGCGAGAGTACAGCTGAATA-3'; vanA-probe, 5'-TET-CTCAGAGGAGCATGACGTATCGGT-BHQI-3'; Unibac fw, 5'-TGGAGCATGTGGTTTAATTCGA-3'; Unibac rv, 5' -TGCGGGACTTAACCCAACA-3'; and Unibac-probe, 5'-TXRed-CACGAGCTGACGACA(AG)CCATGCA-BHQ2-3' (Costa et al., 2005; Klotz et al., 2003; McGregor \& Young, 2000). The probes were labelled with 5carboxyfluorescein (FAM), tetrachloro-6-carboxyfluorescein (TET), Black Hole Quencher 1 (BHQ1) and 2 (BHQ2), or Texas Red (TXRed).

Peripheral blood mononuclear cells (PBMCs). PBMCs were prepared by density-gradient centrifugation (Ficoll Hypaque, $1.077 \mathrm{~g}$ $\mathrm{ml}^{-1}$; Biochrom) from the blood of healthy adult volunteers, as described elsewhere (Bartz et al., 2004).

\section{RESULTS}

\section{Comparison of sample preparation using separator tubes and a Sepsityper kit}

The two different methods of blood culture preparation for subsequent identification by MALDI-TOF MS - separator tubes and a Sepsityper kit - were evaluated. Using the separator tubes, MALDI-TOF MS analysis of positive blood cultures identified $34 \%$ of all bacteria at the species level and $59 \%$ at the genus level (Table 1). Of the Grampositive bacteria, $26 \%$ were correctly identified at the species level and $54 \%$ at the genus level. For Gramnegative bacteria, identification was correct in $50 \%$ at the species level and in $62 \%$ at the genus level. Overall, $38 \%$ of samples were not identified by MALDI-TOF MS. Of the identified bacteria, $100 \%$ were in accordance with the conventional biochemical identification used as the gold standard. These results were based on the following interpretation of score values provided by the BioTyper software: score values of $\geqslant 2.0$ were regarded as correct identification at the species level and values between 1.7 and 1.99 as correct identification at the genus level. Score values of $<1.7$ were termed 'not reliable identification'. Of note, in previous studies presenting a protocol for the preparation of positive blood cultures followed by MALDITOF MS, score values between 1.7 and 1.99 were accepted for identification at the species level if the respective species was stated several times in the list of matching organisms. If we applied this algorithm in the present study, $50 \%$ of Gram-positive bacteria and $62 \%$ of Gram-negative bacteria were correctly identified at the species level.

The new Sepsityper kit from Bruker Daltonics is based on detergent-induced lysis of erythrocytes and leukocytes prior to precipitation of the bacterial pellet. Overall, $85 \%$ of Gram-negative bacteria and $32 \%$ of Gram-positive bacteria were correctly identified at the species level (score value $\geqslant 2.0$ ), whilst $99 \%$ of Gram-negative bacteria and $73 \%$ of Gram-positive bacteria were correctly identified at the genus level (score value $\geqslant 1.7$ ) (Table 2). No misidentification at the species level was observed. If the BioTyper score threshold for species determination was set to $\geqslant 1.7$ as performed in other studies, the identification rates at the species level increased to $99 \%$ for Gramnegative bacteria and $64 \%$ for Gram-positive bacteria. With this algorithm of interpretation, two identifications were incorrect (S. hominis instead of S. epidermidis and $S$. hominis instead of S. haemolyticus).

In summary, compared with the separator tube approach, the Sepsityper kit was especially effective in improving the identification at both genus and species levels for Gramnegative bacteria (from 62 to $99 \%$, and from 50 to $85 \%$, respectively), and at the genus level for Gram-positive bacteria (from 54 to $66 \%$ ); however, no benefit was observed for species identification of Gram-positive cocci. Of note, the overall identification BioTyper score with both methods was significantly higher for Gram-negative bacteria (Sepsityper 2.2 and separator tube 2.0) compared with Gram-positive bacteria (Sepsityper 1.8 and separator tube 1.7).

\section{Interference caused by blood cells}

Repeated preparation of samples with score values of $<1.7$ followed by measurement with MALDI-TOF MS in general did not improve the identification. Interestingly, in a subgroup of blood culture preparations with a lower score value $(<2.0)$, there was a strong correlation with elevated numbers of leukocytes in the original blood samples. To evaluate whether the mass spectra of leukocytes could potentially interfere with those obtained from bacteria, PBMCs were isolated from healthy donors and subjected to the same preparation protocol as the positive blood cultures. Surprisingly, the mass spectra of PBMCs (Fig. 1) did not show any significant overlap when compared with the database containing all mass spectra gained during this study, thus excluding a direct interference of leukocytes with the MALDI-TOF MS method. Strikingly, in all preparations from blood cultures containing high numbers of leukocytes and giving low identification scores, there was only a very small-sized bacterial pellet, or even an invisible bacterial pellet, just prior to the final acidic extraction step. This was surprising, as the Gram stain of the initial positive blood culture showed a high bacterial load. One explanation is that the bacteria were trapped in the gel-like mass of DNA released by leukocytes during the initial lysis step. To investigate this possibility, we removed the leukocytes by an additional low-speed centrifugation step (30 s, $400 \mathrm{~g}$ ) just prior to starting the preparation protocol with the Sepsityper kit. Using this approach, the size of the bacterial pellet increased and the quality of the identifications improved. The preparation of 13 positive blood cultures had to be repeated with such an additional centrifugation 
Table 1. Identification of samples by MALDI-TOF MS after blood culture preparation by gel separator tubes

CoNS, Coagulase-negative staphylococci.

\begin{tabular}{|c|c|c|c|c|c|c|}
\hline \multirow[t]{2}{*}{ Species } & \multirow[t]{2}{*}{ No. samples } & \multicolumn{2}{|c|}{ No. correct } & \multirow{2}{*}{$\begin{array}{l}\text { No. not } \\
\text { identified }\end{array}$} & \multirow[t]{2}{*}{ No. incorrect } & \multirow[t]{2}{*}{ Mean score } \\
\hline & & Score $\geqslant 2.0$ & Score $\geqslant 1.7$ & & & \\
\hline Staphylococcus aureus & 27 & 8 & 13 & 14 & - & 1.9 \\
\hline \multicolumn{7}{|l|}{ CoNS } \\
\hline Staphylococcus capitis & 7 & 3 & 4 & 3 & - & 1.8 \\
\hline Staphylococcus epidermidis & 53 & 9 & 30 & 23 & - & 1.7 \\
\hline Staphylococcus haemolyticus & 4 & 2 & 3 & 1 & - & 2.0 \\
\hline Staphylococcus hominis & 3 & 2 & 3 & - & - & 2.2 \\
\hline Staphylococcus saccharolyticus & 1 & - & - & 1 & - & 1.1 \\
\hline Staphylococcus warneri & 2 & 1 & 1 & 1 & - & 1.8 \\
\hline \multicolumn{7}{|l|}{ Streptococcaceae } \\
\hline Streptococcus pneumoniae & 2 & $2^{*}$ & 2 & - & - & 2.1 \\
\hline Streptococcus gordonii & 2 & - & - & 2 & - & 1.4 \\
\hline Streptococcus anginosus & 1 & - & - & 1 & - & 1.4 \\
\hline Streptococcus intermedius & 1 & - & - & 1 & - & 1.5 \\
\hline \multicolumn{7}{|l|}{ Enterococcaceae } \\
\hline Enterococcus faecalis & 10 & 5 & 6 & 4 & - & 2.3 \\
\hline Enterococcus faecium & 4 & 0 & 2 & 2 & - & 1.7 \\
\hline \multicolumn{7}{|l|}{ Enterobacteriaceae } \\
\hline Citrobacter freundii & 3 & 2 & 3 & - & - & 2.2 \\
\hline Enterobacter cloacae & 2 & - & - & 2 & - & 1.4 \\
\hline Morganella morganii & 1 & 1 & 1 & - & - & 2.0 \\
\hline Klebsiella oxytoca & 2 & - & - & 2 & - & 1.5 \\
\hline Klebsiella pneumoniae & 14 & 10 & 13 & 1 & - & 2.1 \\
\hline Escherichia coli & 19 & 13 & 18 & 1 & - & 2.3 \\
\hline Serratia marcescens & 1 & - & - & 1 & - & 1.6 \\
\hline \multicolumn{7}{|l|}{ Non-fermenters } \\
\hline Acinetobacter baumannii & 1 & - & - & 1 & - & 1.3 \\
\hline Pseudomonas aeruginosa & 9 & 1 & 1 & 8 & - & 1.5 \\
\hline Sphingomonas paucimobilis & 1 & - & - & 1 & - & 1.5 \\
\hline Stenotrophomonas maltophilia & 2 & 1 & 1 & 1 & - & 1.6 \\
\hline \multicolumn{7}{|l|}{ Other species } \\
\hline Lactococcus lactis & 1 & 1 & 1 & - & - & 2.1 \\
\hline Lactobacillus paracasei & 1 & - & - & 1 & - & 1.1 \\
\hline Haemophilus influenzae & 1 & - & 1 & - & - & 1.7 \\
\hline
\end{tabular}

${ }^{\star}$ MALDI-TOF MS differentiation between Streptococcus pneumoniae and Streptococcus mitis is not possible.

step due to no or too small a pellet after the second centrifugation of the Sepsityper protocol. Only two preparations of these positive blood cultures could not be improved using this additional centrifugation step. In these cases, the bacterial pellet was too small to record the MS spectra. In both cases, PCR and the final subculture identified S. aureus.

\section{Differences between spectra from blood cultures and subcultures}

The MS spectra of the two methods were compared with spectra obtained from the same bacteria after cultivation on blood agar plates and in brain-heart infusion broth or in pure blood culture medium (i.e. in the absence of any blood). Compared with the other methods, additional peaks were detectable in the preparations obtained directly from blood cultures: a group of eight to 12 peaks in the lower mass range between 2000 and $4000 \mathrm{Da} / z$ and one or more significant peaks $>7000 \mathrm{Da} / z$. Therefore, additional peaks may be caused by blood components though not by resins or other compounds within the blood culture medium (Fig. 1).

\section{Supplementing the workflow for Gram-positive cocci by PCR}

As mentioned above, identification of bacteria by MALDITOF MS from positive blood cultures was especially successful for Gram-negative bacteria but showed problems 
Table 2. Identification of samples by MALDI-TOF MS after blood culture preparation using the Sepsityper kit

\begin{tabular}{|c|c|c|c|c|c|c|}
\hline \multirow[t]{2}{*}{ Species } & \multirow[t]{2}{*}{ No. samples } & \multicolumn{2}{|c|}{ No. correct } & \multirow{2}{*}{$\begin{array}{c}\text { No. not } \\
\text { identified }\end{array}$} & \multirow[t]{2}{*}{ No. incorrect } & \multirow[t]{2}{*}{ Mean score } \\
\hline & & Score $\geqslant 2.0$ & Score $\geqslant 1.7$ & & & \\
\hline \multicolumn{7}{|l|}{ CoNS } \\
\hline Staphylococcus capitis & 1 & - & 1 & - & - & 1.8 \\
\hline Staphylococcus hominis & 6 & 1 & 3 & 3 & - & 1.9 \\
\hline Staphylococcus lugdunensis & 1 & - & 1 & - & - & 1.7 \\
\hline \multicolumn{7}{|l|}{ Streptococcaceae } \\
\hline Streptococcus agalactiae & 1 & 1 & 1 & 1 & - & 2.2 \\
\hline Streptococcus pyogenes & 2 & 1 & 2 & - & - & 1.7 \\
\hline \multicolumn{7}{|l|}{ Enterococcaceae } \\
\hline Enterobacter aerogenes & 2 & 2 & 2 & - & - & 2.6 \\
\hline Enterobacter hormaechei & 1 & 1 & 1 & - & - & 2.4 \\
\hline Enterobacter cloacae & 3 & 2 & 3 & - & - & 2.2 \\
\hline Proteus mirabilis & 1 & 1 & 1 & - & - & 2.5 \\
\hline Klebsiella oxytoca & 1 & 1 & 1 & - & - & 2.4 \\
\hline Klebsiella pneumoniae & 4 & 2 & 4 & - & - & 2.1 \\
\hline Escherichia coli & 27 & 25 & 27 & - & - & 2.3 \\
\hline Salmonella paratyphi B & 1 & $1 \dagger$ & $1 \dagger$ & - & - & 1.9 \\
\hline Serratia marcescens & 2 & 2 & 2 & - & - & 2.1 \\
\hline \multicolumn{7}{|l|}{ Non-fermenters } \\
\hline Acinetobacter baumannii & 1 & - & 1 & - & - & 1.8 \\
\hline Clostridium innocuum & 1 & 1 & 1 & - & - & 2.0 \\
\hline Pediococcus pentosaceus & 1 & - & 1 & - & - & 1.8 \\
\hline
\end{tabular}

${ }^{\star}$ BioTyper score of 1.78 and 1.82, and two different staphylococci within the first three proposals of the results. $\nmid$ MALDI-TOF MS differentiation of Salmonella species is possible only at the genus level.

for Gram-positive bacteria. In the routine diagnostics of the microbiological laboratory at the University Hospital of Heidelberg, about $80 \%$ of the positive blood culture bottles contained Gram-positive grape-like clustered cocci. Among these bacteria, the identification of S. aureus and the detection of possible resistance genes are of outstanding importance for an early adequate treatment. The resistance of staphylococci against all $\beta$-lactam antibiotics (meticillin resistance) is caused by the expression of a single gene, mecA, which encodes the penicillin-binding protein 2a (PBP2a) (Katayama et al., 2000). Thus, detection of the mecA cassette by PCR enables identification of the most important resistance mechanism known for staphylococci. Therefore, a PCR detecting both $S$. aureus (Klotz et al., 2003) and the meticillin resistance gene in
DNA isolated directly from positive blood cultures was established and included in the standard diagnostic protocol. During the observation period, S. aureus was detected in 66/ 761 positive blood culture bottles. Of these, $14 \mathrm{~S}$. aureus isolates turned out to be meticillin resistant. The sensitivity and specificity for $S$. aureus as well as for mecA detection by direct PCR were $100 \%$, as confirmed by conventional testing of the subcultured bacteria.

According to current guidelines, calculated antibiotic treatment of bloodstream infections includes glycopeptide antibiotics for coverage of Gram-positive bacteria. Vancomycin-resistant enterococci (VRE) have emerged as an increasing problem in the modern healthcare system. 


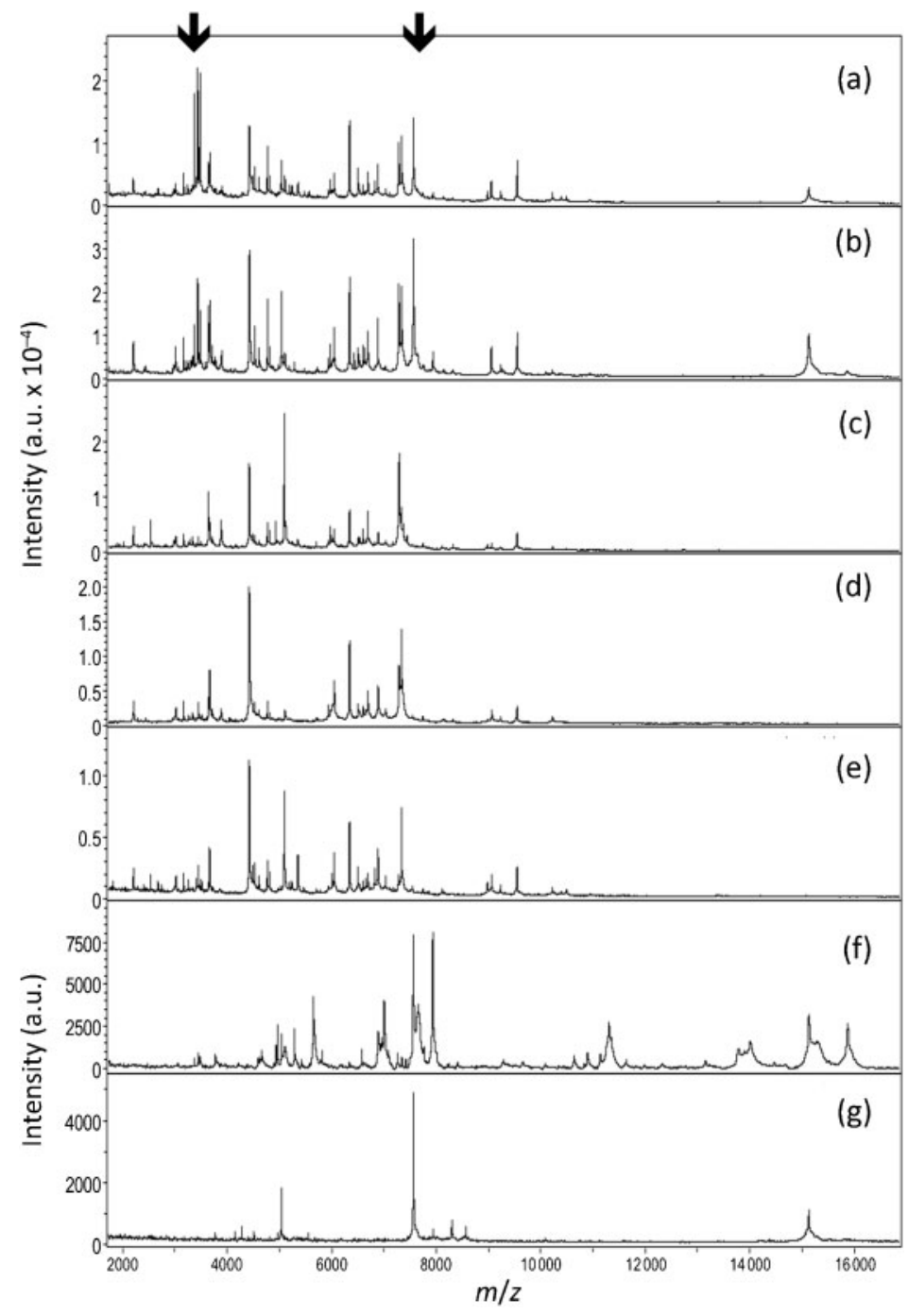

Fig. 1. Comparison of mass spectra for a single, model Enterococcus faecium strain (patient isolate) generated by three different MALDI-TOF MS determinations from Columbia agar with $5 \%$ sheep blood (a), after preparation from blood cultures by separator gel (b), after preparation from blood cultures by the Sepsityper kit (c), from inoculated blood culture medium with the isolated E. faecium strain (d), from inoculated brain heart infusion broth (e), from isolated PBMCs (f) and from isolated erythrocytes (g). The arrowed peaks appeared only in preparations from positive blood cultures. a.u., Arbitrary units.

Bloodstream infection with VRE, especially in immunocompromised patients, is a threat (Avery et al., 2005). Appropriate adjustment of the antimicrobial treatment should be performed as early as possible. A PCR for detection of the most frequent genes responsible for VRE ( vanA and $v a n B$ ) was included in the routine diagnostic course for positive blood cultures with Gram-positive cocci. During the observation period, one vanA-positive and four $v a n B$-positive enterococci were detected in the blood cultures, resulting in a sensitivity and specificity of $100 \%$.

\section{DISCUSSION}

The aim of this study was to evaluate and optimize the efficacy of direct identification of bacteria from positive blood culture bottles by MALDI-TOF MS using the new Sepsityper kit and a separator gel-based method. The first part of the study showed that, in accordance with the results of other studies, direct identification of bacteria from positive blood cultures by MALDI-TOF MS is a fast and reliable method. A major problem of the currently published preparation protocols for identification of bacteria directly from blood culture is that the BioTyper score values are lower compared with identification from culture plates, meaning a less reliable result. In order to increase identification rates, previously studies have accepted results with lower score values (Moussaoui et al., 2010; Szabados et al., 2011). In the present publication, direct identification by MALDI-TOF MS of bacteria from positive blood culture bottles showed much better results for Gram-negative than for Gram-positive bacteria, independent of the preparation protocol used. The overall quality of identification with the Sepsityper kit was superior to that following preparation with gel separator tubes. In particular, the identification of non-fermenting Gram-negative rods, including Pseudomonas aeruginosa but also the group of Enterobacteriaceae, gave more reliable results when prepared with the Sepsityper kit. Due to excellent direct MALDI-TOF MS identification of Gramnegative bacteria with the Sepsityper kit, accepting lower score values was not necessary. The limitation of the 
present study is that the two methods were not compared directly with the same samples, so that the investigated bacteria were divergent.

The major problem of both sample preparation methods for the direct identification of bacteria from positive blood cultures by MALDI-TOF MS was poor identification of the Gram-positive grape-like clustered cocci. As differentiation of $S$. aureus from CoNS in positive blood cultures is of high clinical importance, considerable effort has been made to improve the preparation method. An important difference between Gram-negative and Gram-positive bacteria is the composition of the bacterial cell wall. For better disruption of the peptidoglycan layer of the Grampositive bacterial cell wall, it has been proposed that the bacteria are treated with ultrasound. However, treatment of the final bacterial lysates in acetonitrile and formic acid with ultrasound in the present study improved the result of the identification in only a few cases (data not shown). Moussaoui et al. (2010) suggested also accepting bacterial identification with a BioTyper score of $<1.7$ if four successive proposals with a score $\geqslant 1.4$ were identical. This is in agreement with the results obtained in the present study. Such alteration of the threshold improved correct identification by $\sim 8 \%$ for the preparation with gel separator tubes and by $6 \%$ with the Sepsityper kit (data not shown). Thus, decreasing the threshold of the Bruker score for the MALDI-TOF MS identification of bacteria at the species level at least down to 1.7 if the first four successive proposals of the biotype are identical appears to be an acceptable approach to improve the identification rate of direct MALDI-TOF MS analysis for Gram-positive grape-like clustered cocci. Both misidentified staphylococci (Table 2) in this study had two different staphylococci within the first three proposals of the results, so the identification would have been excluded if the algorithm had been applied. In contrast to Moussaoui et al. (2010), we instead propose performing PCR and subcultures to guarantee sufficient specificity.

In the present study, the overall identification rate of Gram-positive grape-like clustered cocci still remained less than $70 \%$ for both methods. Considering that in the daily routine of the microbiological laboratory, a large proportion of positive blood cultures consists of Gram-positive grape-like clustered cocci (up to $80 \%$ ), identification rates for the latter of only $70 \%$ are unacceptably low. For the fast identification of S. aureus, we therefore propose performing a PCR directly from all positive blood cultures in the case of Gram-positive cocci. The final differentiation of CoNS should be performed after subculture with conventional methods. The direct PCR can be considered extremely valuable, as it provides additional information on susceptibility towards antibiotics by allowing the detection of the resistance genes mecA, vanA and vanB.

Given the described advantages of the PCR, this technology could be used for detecting pathogen DNA directly in the blood of septic patients. Several commercial systems are available for analysis of whole blood within 6-7 h (Dark et al., 2009). The question arises as to why MALDI-TOF MS in combination with PCR from positive blood cultures should be used and not PCR alone, performed directly from the blood without using blood cultures. Commercially available multiplex PCR approaches are time-intensive, expensive and cover only a restricted pathogen spectrum, albeit with high sensitivity. As only limited information on susceptibility towards antibiotics can be achieved by direct PCR, cultivation of the bacteria for susceptibility testing always needs to be performed additionally. In the current project, the blood culture flasks containing Enterobacteriaceae, enterococci and staphylococci were on average detected as positive after $15 \mathrm{~h}$, whereas CoNS needed $22 \mathrm{~h}$ on average (data not shown). Gram staining and the MALDI-TOF MS identification need about $1 \mathrm{~h}$. The PCRs for $f e m B, m e c A$, van $A$ and $v a n B$ take another $2 \mathrm{~h}$. Thus, the results are expected to be available within 1 day, roughly $12-$ $24 \mathrm{~h}$ later than the multiplex PCR performed directly from the blood.

As a final workflow for the routine processing of positive blood cultures, a flowchart is proposed, as shown in Fig. 2. The initial Gram stain decides on the further processing of the blood culture. Gram-negative bacteria, Gram-positive cocci in chains and Gram-positive rods are subjected to preparation using the Sepsityper kit followed by MALDITOF MS. AST is performed for all blood cultures preliminarily by an agar diffusion test directly from the positive blood cultures (Bennett \& Sharp, 2008). After differentiation by MALDI-TOF MS, the final susceptibility is determined by conventional AST methods. For Gramnegative rods, AST can also be performed directly from the blood culture using Vitek2 and the protocol of Funke \& Funke-Kissling (2004) in parallel with the preliminary testing. For Gram-positive cocci, preliminary AST is performed by agar diffusion testing. The identification and final susceptibility testing are performed from the subculture. For all blood cultures containing Gram-positive cocci, a combined direct PCR should be performed for the detection of $f e m B$, mecA, vanA and vanB.

Theoretically, all positive blood cultures could be analysed with our proposed workflow in order to achieve an identification result on the day of positivity. The handson time was about $20-25 \mathrm{~min}$ for five samples for the Sepsityper kit, and $45 \mathrm{~min}$ for five samples for the PCR. If direct identification is restricted to Gram-negative bacteria, Gram-positive cocci in chains and Gram-positive rods, the total number of blood culture preparations for direct MALDI-TOF MS identification remains manageable. Depending on the working hours of the laboratory, it is possible to analyse a large number of positive blood cultures on the day of positivity - theoretically all of them.

In summary, MALDI-TOF MS is a fast and reliable technique for identification of bacteria in positive blood cultures. The Sepsityper kit analysed here gave good and reliable results for Gram-negative bacteria and may be 


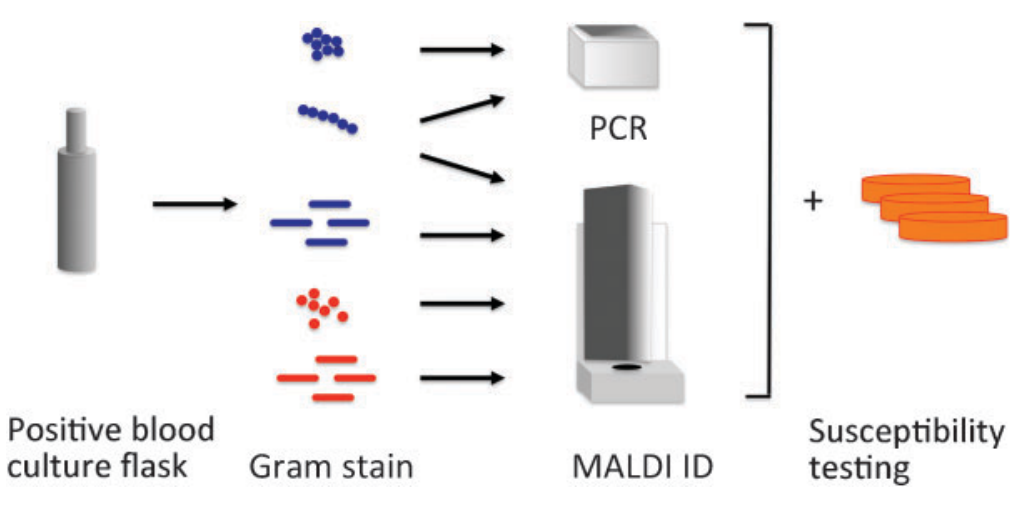

Fig. 2. Workflow for processing of positive blood cultures for identification and antibiotic susceptibility testing of bacteria from bloodstream infections. The initial Gram stain decides on the further processing of the blood culture. Gram-negative bacteria (red), Grampositive cocci in chains and Gram-positive rods are subjected to preparation using the Sepsityper kit followed by MALDI-TOF MS. For blood cultures containing Gram-positive cocci, a combined direct $\mathrm{PCR}$ is performed for the detection of $f e m B, \operatorname{mec} A$, vanA and vanB. AST is performed for all blood cultures preliminarily by an agar diffusion test directly from positive blood cultures. helpful for identification of Gram-positive rods. The combination of MALDI-TOF MS with the PCR described in the current study enables a microbiological laboratory quickly to provide essential information for antimicrobial therapy of bloodstream infections. The results are available up to 2 days earlier than with current routine procedures, thus improving the therapy for critically ill patients.

\section{REFERENCES}

Avery, R., Kalaycio, M., Pohlman, B., Sobecks, R., Kuczkowski, E., Andresen, S., Mossad, S., Shamp, J., Curtis, J. \& other authors (2005). Early vancomycin-resistant enterococcus (VRE) bacteremia after allogeneic bone marrow transplantation is associated with a rapidly deteriorating clinical course. Bone Marrow Transplant 35, 497-499.

Bartz, H., Mendoza, Y., Gebker, M., Fischborn, T., Heeg, K. \& Dalpke, A. (2004). Poly-guanosine strings improve cellular uptake and stimulatory activity of phosphodiester $\mathrm{CpG}$ oligonucleotides in human leukocytes. Vaccine 23, 148-155.

Bennett, K. \& Sharp, S. E. (2008). Rapid differentiation of methicillin-resistant Staphylococcus aureus and methicillin-susceptible Staphylococcus aureus from blood cultures by use of a direct cefoxitin disk diffusion test. J Clin Microbiol 46, 3836-3838.

Bochud, P. Y., Bonten, M., Marchetti, O. \& Calandra, T. (2004). Antimicrobial therapy for patients with severe sepsis and septic shock: an evidence-based review. Crit Care Med 32 (Suppl.), S495S512.

Christner, M., Rohde, H., Wolters, M., Sobottka, I., Wegscheider, K. \& Aepfelbacher, M. (2010). Rapid identification of bacteria from positive blood culture bottles by use of matrix-assisted laser desorption-ionization time of flight mass spectrometry fingerprinting. J Clin Microbiol 48, 1584-1591.

Costa, A. M., Kay, I. \& Palladino, S. (2005). Rapid detection of mecA and $n u c$ genes in staphylococci by real-time multiplex polymerase chain reaction. Diagn Microbiol Infect Dis 51, 13-17.

Dark, P. M., Dean, P. \& Warhurst, G. (2009). Bench-to-bedside review: the promise of rapid infection diagnosis during sepsis using polymerase chain reaction-based pathogen detection. Crit Care 13, 217.

Emonet, S., Shah, H. N., Cherkaoui, A. \& Schrenzel, J. (2010). Application and use of various mass spectrometry methods in clinical microbiology. Clin Microbiol Infect 16, 1604-1613.
Ferreira, L., Sánchez-Juanes, F., Porras-Guerra, I., Garcia-Garcia, M. I., García-Sánchez, J. E., González-Buitrago, J. M. \& MuñozBellido, J. L. (2011). Microorganisms direct identification from blood culture by matrix-assisted laser desorption/ionization time-of-flight mass spectrometry. Clin Microbiol Infect 17, 546-551.

Funke, G. \& Funke-Kissling, P. (2004). Use of the BD PHOENIX Automated Microbiology System for direct identification and susceptibility testing of Gram-negative rods from positive blood cultures in a three-phase trial. J Clin Microbiol 42, 1466-1470.

Garnacho-Montero, J., Ortiz-Leyba, C., Herrera-Melero, I., AldabóPallás, T., Cayuela-Dominguez, A., Marquez-Vacaro, J. A., CarbajalGuerrero, J. \& Garcia-Garmendia, J. L. (2008). Mortality and morbidity attributable to inadequate empirical antimicrobial therapy in patients admitted to the ICU with sepsis: a matched cohort study. J Antimicrob Chemother 61, 436-441.

Ibrahim, E. H., Sherman, G., Ward, S., Fraser, V. J. \& Kollef, M. H. (2000). The influence of inadequate antimicrobial treatment of bloodstream infections on patient outcomes in the ICU setting. Chest 118, 146-155.

Katayama, Y., Ito, T. \& Hiramatsu, K. (2000). A new class of genetic element, Staphylococcus cassette chromosome mec, encodes methicillin resistance in Staphylococcus aureus. Antimicrob Agents Chemother 44, 1549-1555.

Klotz, M., Opper, S., Heeg, K. \& Zimmermann, S. (2003). Detection of Staphylococcus aureus enterotoxins A to D by real-time fluorescence PCR assay. J Clin Microbiol 41, 4683-4687.

Kumar, A., Roberts, D., Wood, K. E., Light, B., Parrillo, J. E., Sharma, S., Suppes, R., Feinstein, D., Zanotti, S. \& other authors (2006). Duration of hypotension before initiation of effective antimicrobial therapy is the critical determinant of survival in human septic shock. Crit Care Med 34, 1589-1596.

Kumar, A., Ellis, P., Arabi, Y., Roberts, D., Light, B., Parrillo, J. E., Dodek, P., Wood, G., Kumar, A. \& other authors (2009). Initiation of inappropriate antimicrobial therapy results in a fivefold reduction of survival in human septic shock. Chest 136, 1237-1248.

McGregor, K. F. \& Young, H.-K. (2000). Identification and characterization of vanB2 glycopeptide resistance elements in enterococci isolated in Scotland. Antimicrob Agents Chemother 44, 2341-2348.

Mellmann, A., Bimet, F., Bizet, C., Borovskaya, A. D., Drake, R. R., Eigner, U., Fahr, A. M., He, Y., Ilina, E. N. \& other authors (2009). High interlaboratory reproducibility of matrix-assisted laser desorption ionization-time of flight mass spectrometry-based species identification of nonfermenting bacteria. J Clin Microbiol 47, 37323734 . 
Moussaoui, W., Jaulhac, B., Hoffmann, A. M., Ludes, B., Kostrzewa, M., Riegel, P. \& Prévost, G. (2010). Matrix-assisted laser desorption ionization time-of-flight mass spectrometry identifies $90 \%$ of bacteria directly from blood culture vials. Clin Microbiol Infect 16, 16311638.

Stevenson, L. G., Drake, S. K. \& Murray, P. R. (2010). Rapid identification of bacteria in positive blood culture broths by matrix-assisted laser desorption ionization-time of flight mass spectrometry. J Clin Microbiol 48, 444-447.

Szabados, F., Michels, M., Kaase, M. \& Gatermann, S. (2011). The sensitivity of direct identification from positive BacT/ALERT (bioMérieux) blood culture bottles by matrix-assisted laser desorption ionization time-of-flight mass spectrometry is low. Clin Microbiol Infect 17, 192-195. 